\title{
Desarrollo del pensamiento complejo y la enseñanza de la Historia: Una experiencia de aprendizaje en una escuela primaria indígena
}

\author{
Development of complex thinking and teaching of History: A learning experience in the Indigenous \\ Primary School \\ L. Lizbeth Tzongua-Demha ${ }^{a}$, Antonia Olguín-Neria ${ }^{b}$
}

\begin{abstract}
:
This contribution shows the ideas and perspectives on the development of complex thinking about the teaching of history within the Indigenous Primary School. At the same time, it provides a living experience on the teaching of History within the teaching practice. This complex thought is of vital importance both socially and during the process of teaching History at this educational level. For this reason, innovation as part of the teaching of History, will allow complex thinking to be developed and, in this way, students will be able to acquire knowledge and solid bases in the construction of their learning process, History as a subject has been a fundamental part in the development of societies. It allows to form analytical and reflective individuals that will take appropriate and relevant decisions for social development.
\end{abstract}

\section{Keywords:}

Complex thinking, Teaching, History, Indigenous education

\section{Resumen:}

Esta aportación aborda ideas y perspectivas sobre el desarrollo del pensamiento complejo y la enseñanza de la Historia dentro de la Escuela Primaria Indígena, de igual manera aporta una experiencia vivida sobre la impartición de la asignatura de Historia dentro de la práctica docente. El pensamiento complejo toma vital importancia tanto en lo social como dentro del proceso de enseñanza de la Historia en este nivel educativo. Por tal motivo, la innovación como parte de enseñanza de la Historia permitirá que se desarrolle el pensamiento complejo y de esta manera, los alumnos podrán adquirir conocimientos y bases sólidas en la construcción de su proceso de aprendizaje. La Historia, como una de las asignaturas de la educación básica, ha sido parte fundamental para el desarrollo de las sociedades, permite formar individuos analíticos y reflexivos en torno a la toma de decisiones propicias y pertinentes para el desarrollo social.

\section{Palabras Clave:}

Pensamiento complejo, Enseñanza, Historia, Educación indígena

Recibido: 20 de junio de 2018

Dictaminado: 28 de agosto de 2018

Segunda versión: 20 de septiembre de 2018

Aceptado: 4 de octubre de 2018

\footnotetext{
${ }^{a}$ Estudiante del séptimo semestre de la Licenciatura en Primaria Intercultural Bilingüe. Escuela Normal Valle del Mezquital. Progreso de Obregón, Hidalgo, México. Email: liztzongua@gmail.com

b Docente-Investigadora. Escuela Normal Valle del Mezquital. Progreso de Obregón, Hidalgo, México. Email: tonanery@ hotmail.com
} 


\section{Introducción}

La enseñanza de la Historia es indispensable para el conocimiento del ser humano al vivir en sociedad; si damos una breve mirada desde los tiempos remotos hasta los días actuales, advertimos que los motivos que hoy nos mueven a enseñar la Historia no difieren sustancialmente de los fines que animaron a nuestros antepasados, para dar a conocer la historia propia y la de otros pueblos. Esto para hacerlos conscientes de que son parte de la gran corriente de la historia humana, de un proceso que se inició hace miles de años y con cambios constantes. Al respecto, Bracho (2009: 254) señala que "la Historia es impensable sin la memoria", brinda una perspectiva alentadora de las vivencias del pasado, las consecuencias que han traído al presente y el desarrollo de nuestro futuro, por lo que resulta importante estudiar los sucesos históricos que dieron origen a las sociedades actuales.

Bajo las premisas anteriores, en este ensayo se aborda el desarrollo del pensamiento complejo, los propósitos que tiene a la enseñanza de la Historia en la Primaria Indígena, así como, el papel que juega el pensamiento complejo en la enseñanza de la Historia, a partir de la recuperación de una experiencia de enseñanza en la práctica docente en la asignatura de la Historia con un grupo de quinto grado, en una escuela primaria indígena. El trabajo cierra con algunas conclusiones, que toman como base la experiencia en el aula y sobre todo, los beneficios que trae consigo el desarrollo del pensamiento complejo dentro de la enseñanza de la Historia.

\section{Desarrollo del pensamiento complejo}

La teoría del pensamiento complejo dice que la realidad es comprendida y contemplada simultáneamente desde todas las perspectivas posibles, de esta manera le damos resignificación para poder estudiar de forma compleja; es decir, de una manera global, no puede ser fragmentada; porque limita el campo de acción del conocimiento, de ahí que recurrir a la complejidad permite entender el mundo que nos rodea. El pensamiento complejo hace referencia a la capacidad de interconectar distintas dimensiones de lo real, pero también debemos cuestionar: ¿qué es pensar?, pensar es una actividad que realizamos de manera natural y espontánea. A cada instante de nuestra vida diaria, todas las personas desarrollamos los factores que intervienen dentro del pensamiento; el "Yo" como sujeto pensante al desarrollar un proceso de pensar.

Realizar un proceso de pensamiento implica una expresión de lo que nosotros pensemos. Mediante el pensamiento complejo el individuo recurre a la parte humanista que es un parteaguas para tener la necesidad de buscar soluciones a las crisis de la sociedad, a través de lo que se define como un método de pensamiento para comprender la naturaleza, la sociedad en la que nos vemos envueltos y de esta manera, poder reorganizar una vida en sociedad. Desde esta perspectiva, el pensamiento complejo permite interconectar distintas dimensiones de lo real, se basa en el análisis, la desunión y la simplicidad.

De allí [es decir, de esta problemática], se esbozan las dos grandes finalidades ético-políticas del nuevo milenio: establecer una relación de control mutuo entre la sociedad y los individuos por medio de la democracia y concebir la Humanidad como comunidad planetaria. La educación debe no sólo contribuir a una toma de conciencia de nuestra Tierra-Patria, sino también permitir que esta conciencia se traduzca en la voluntad de realizar la ciudadanía terrenal (Morín, 1999: 7).

Respecto a lo que menciona Morín, la educación va más allá de formarnos y sentirnos parte de esta sociedad. Ésta no debe limitarse a eso, sino a enseñar a los educandos a pensar, a razonar, a buscar explicaciones de diversos fenómenos y/o sucesos. El estudio de lo complejo ha impactado significativamente al ámbito de la educación, la manera de ver de la sociedad, las diversas formas de organización social. Aunado a todo esto, se busca comprender cada fenómeno, al considerar el pensamiento complejo para comprender los procesos de la vida social en el que nos desarrollamos, que implica hacer frente a la realidad y encontrar soluciones que nos permitan vivir en armonía. Lleva a despertar el interés de los individuos, porque desarrolla la capacidad de asombro, imaginación e interés de forma sustancial por lo desconocido, al brindar una aproximación de lo que se desea conocer y de esta manera investigar a través de la inteligencia y la síntesis de significados.

\section{Enseñanza de la Historia}

Cuestionar ¿para qué sirve la Historia en nuestros alumnos de la escuela primaria indígena?, lleva a voltear la mirada al pasado y encontrar posibles respuestas multidimensionales a los acontecimientos. Los niños deben comprender lo que acontece en el mundo en donde se desenvuelven, conocer los cambios y las causas de los sucesos que dieron origen a la situación actual, desde el ámbito social, político, económico y cultural. Para ellos, el presente es lo único que importa; sin embargo, lo que hace que la enseñanza de la Historia adquiera relevancia es porque aporta conocimientos, desarrolla habilidades y valores que están relacionados con el proceso de conformación de él como persona, pero también de su "Yo", como parte de una comunidad y sociedad en lo particular. Esto facilita la búsqueda de respuestas a las interrogantes del mundo actual. Los niños parten de interrogantes y de premisas para poder conocer el entorno en el que se desenvuelven; el 
cuestionar les permite resolver sus dudas dentro y fuera del aula. Así, el niño busca indagar sobre las dudas que posee; conocer el pasado sirve para explicar el presente y ofrecer una perspectiva que ayuda a su comprensión. Sánchez (2005: 60) explica que: "la Historia es científica en la medida que comienza por hacer preguntas".

El propósito principal de la enseñanza de la Historia, en la escuela primaria indígena, se orienta a lograr que los alumnos desarrollen el pensamiento y la conciencia histórica; así, vislumbren lo que sucede en las sociedades contemporáneas y participen en acciones de beneficio social, de manera responsable e informada. Mediante el aprendizaje de la Historia los alumnos comprenden los hechos del pasado, explican sus causas-consecuencias, los cambios, continuidades y la influencia en el presente. Es decir, permite comprender los problemas sociales, crear explicaciones y darle importancia a los acontecimientos que son parte de la realidad cotidiana, a usar la información para poder desarrollar una conciencia ciudadana.

Se reconoce a la Historia como una ciencia que estudia las transformaciones que experimentan las sociedades a lo largo del tiempo y que no se posee verdades absolutas, las ideas están sujetas a los hallazgos o explicaciones, puesto que el conocimiento histórico está en constante revisión (Lima, Bonilla y Arista, 2010: 3).

Uno de los propósitos de la enseñanza de la Historia en educación básica está dirigido a que los niños aprendan a pensar históricamente, e implica partir desde lo que viven actualmente y comprender cómo es que nos situamos en el contexto social. En diversas ocasiones, como individuos, nos preguntamos: ¿para qué aprender Historia?, algunos tienen perspectivas y opiniones diversas, no obstante, sabemos desde las nociones de la vida cotidiana, que el que desconoce la historia está condenado a repetirla. Esta expresión, resulta acertada, en virtud de que se considera importante el hecho de que si conocemos el pasado podemos analizar y reflexionar sobre lo que acontece en la actualidad, y de esta manera, realizar acciones y emitir juicios de valor para no repetir las mismas acciones que podrían repercutir en un futuro. Todos los individuos somos seres sociales, que de acuerdo con Peter Berger y Thomas Luckmann (1968), transitamos por procesos de socialización que son los que nos van conformado como personas pertenecientes a una determinada sociedad que se va construyendo de acuerdo con las experiencias que experimentamos en nuestras vidas. Por tanto, no se concibe que un individuo no sea parte de una sociedad o que solamente trabaje de manera individual, es por medio de la sociedad que se aprenden algunas acciones que realizamos. Esto es, los autores mencionados refieren que:
Todo individuo nace dentro de una estructura social objetiva en la cual encuentra a los otros significantes que están encargados de su socialización y que les son impuestos. Las definiciones que los otros significantes hacen de la situación del individuo le son presentadas a éste como realidad objetiva. De este modo, él nace no sólo dentro de una estructura social objetiva, sino también dentro de un mundo social objetivo (Berger y Luckmann, 1968: 166).

Conocer la Historia de nuestro origen, de nuestra familia, de la comunidad, de nuestro contexto, nos ayuda a entender quiénes somos, el porqué de la situación actual, sí desconocemos la Historia nos desconocemos a nosotros mismos. Sin duda alguna, nos brinda identidad y nos da un sentido de pertenencia como parte de una sociedad en la cual nos desarrollamos, de ello que la enseñanza de la historia "es importante por ser la memoria de la humanidad que nos introduce en la evolución de la civilización y los logros del género humano" (SEP, 2011: 11). En este sentido, el papel del docente es relevante, ya que de acuerdo con Sebastián Plá, (2011), el profesor es el sujeto central del proceso educativo y como tal, requiere de conocimientos disciplinares y didácticos específicos, así como principios éticos de comportamiento muy particulares. Luego entonces enseñar Historia, no es para que los alumnos aprendan fechas, sino favorecer la adquisición de la comprensión de los sucesos históricos que han ocurrido a lo largo del tiempo, debe buscar estrategias para que los niños construyan nociones sociales y desarrollen capacidades cognitivas dentro del aula.

[...] no debe ser la de inculcarles a nuestros alumnos una serie de verdades establecidas sobre el pasado, sino la de alimentar sus mentes, no sólo con conocimientos históricos concretos para que puedan operar con ellos, sino contribuyendo a formar un sentido crítico que les lleve a entender que son ellos quienes deben utilizar este aprendizaje para juzgar, con la experiencia adquirida, el paisaje social que les rodea, sin admitir que se les diga que es el producto de una evolución lógica, natural e inevitable, y que debe, por tanto, aceptarse sin discusión, crítica ni resistencia (Fontana, 2008:10 en Arteaga, 2014: 121).

Es necesario que conozca los elementos conceptuales propios del contenido que se va a enseñar y aprender, al procurar que en el alumno produzca una apropiación del objeto de estudio. Para enseñar Historia, como plantea en la actualidad la Secretaría de Educación Pública, se requiere del dominio de los contenidos, análisis, reflexión, crítica formativa y de un rigor lógico para una búsqueda de la verdad, para que los conocimientos que adquieran los alumnos les ayuden a comprender y actuar en su entorno.

El centro y el referente fundamental del aprendizaje es el estudiante, porque desde etapas tempranas se 
requiere generar su disposición y capacidad de continuar aprendiendo a lo largo de su vida, desarrollar habilidades superiores del pensamiento para solucionar problemas, pensar críticamente, comprender y explicar situaciones desde diversas áreas del saber, manejar información, innovar y crear en distintos órdenes de la vida (SEP, 2011: 26).

Para enseñar Historia el docente tiene que despertar el interés de los estudiantes y persuadirlos de la utilidad e importancia de la historia. Ese saber, acompaña a los hombres desde el inicio de su vida. La memoria de sus experiencias y descubrimientos, permite la acumulación de conocimiento, por no sólo eso, sino su avance continuo en ese devenir histórico. "No sólo soy objeto de la Historia, sino también su sujeto. En el mundo de la Historia, de la cultura, de la política, no constato para adaptarme sino para cambiar" (Freire, 1997: 100). Esto lleva a formarnos una idea que un individuo es parte de la Historia y parte de una sociedad en constante cambio, en donde todos interactuamos. En la escuela, los alumnos deben conocer los acontecimientos de los que fueron parte las personas que vivieron antes que ellos, conocer sus formas de ver la vida, así como los aportes que sirvieron para que hoy tengamos la realidad que nos está tocando vivir. Por eso, es importante ver a la Historia no sólo como una secuencia cronológica de hechos importantes para la humanidad, sino también identificarla como la ciencia cuyo objeto de estudio es el hombre, y lograr una conciencia histórica que repercuta en la vida cotidiana. Sin el estudio de la Historia, nuestras sociedades pudieron haber perdido su esencia, los pueblos conocen el proceso que ha gestado su desarrollo y desenvolvimiento, esto ha creado bases para tomar otros rumbos dentro de la sociedad, desde las antiguas hasta las actuales.

Es necesario que los docentes se preparen para motivar a sus alumnos a leer y conocer los antecedentes de la vida que hoy nos tocó vivir. La enseñanza de la Historia direcciona a una perspectiva para que ellos aprendan, porque somos parte de ella, porque considera las actitudes y valores de una sociedad; una sociedad cambiante y globalizada en la que es pertinente considerar dichos cambios para que no repetir los errores del pasado. Los seres humanos somos parte minúscula de la Historia, nuestros antepasados han sido partícipes de ella, somos producto de las interacciones pasadas entre individuos.

En la educación básica se promueve la enseñanza de una historia formativa que analiza el pasado para encontrar explicaciones del presente y entender cómo las sociedades actuaron ante determinadas circunstancias y esto permita vislumbrar un futuro mejor. Para ello se considera necesario dejar atrás una enseñanza centrada en la repetición de hechos, lugares y personajes históricos, y encaminarse a un aprendizaje que reflexione sobre el pasado para responsabilizarse y comprometerse con el presente (SEP, 2018: 384).

Trabajar la historia desde este planteamiento considera al aprendizaje como un proceso activo de descubrimiento y de indagación, requiere trabajar en las aulas observaciones racionales, entre las que destacan la capacidad de clasificar, comparar, analizar, describir, inferir, explicar, memorizar, ordenar las ideas, etcétera. La historia es una disciplina que sin análisis crítico no existiría. Por lo tanto, enseñar y aprender historia es estimular el pensamiento. El estudio de la Historia se basa en métodos de análisis, y se considera necesaria la aplicación del método histórico, cuyas fases se pueden esquematizar de la siguiente forma (SEP, 2011):

- Ante una interrogante o enigma se procede a intentar conocer qué es aquello que otros han dicho o han respondido.

- Si las respuestas no son lo suficientemente satisfactorias, se recurre a fuentes primarias sobre el tema.

- Con el análisis de fuentes es posible plantear hipótesis que intenten explicar o resolver las cuestiones planteadas.

- Para validar o probar las hipótesis se tienen que buscar nuevas fuentes.

- Clasificar para poder ser utilizadas.

- Las fuentes han de ser contrastadas y evaluadas críticamente.

- Al final del proceso se elaboran conclusiones de la investigación destacando los puntos débiles, es decir, aquello que no ha podido ser demostrado o resuelto.

La enseñanza de la Historia, con responsabilidad social, tendrá que potenciar en los alumnos el sentido de identidad, al sentirse parte de la Historia y de la vida de su país, porque conoce de sus costumbres, tradiciones y es defensor de esos valores que lo identifican. La responsabilidad social del docente radica en despertar el interés del alumno hacia el pasado, esto facilita la comprensión del presente y reflexionar sobre su proyección futura, a partir de los hechos, procesos y fenómenos con el protagonismo de los actores de la Historia. Esto permitirá tener una identidad propia y visualizar el papel que le corresponde en la sociedad como generación adulta. Preparar a los niños para la toma de decisiones, al comprender desde la Historia los problemas sociales para formar criterios, actitudes y valores relacionados con la vida-ciudadana, implica una toma de postura que representa un cambio en el rumbo de nuestro país. El ciudadano ideal en la sociedad del conocimiento es aquel que se caracteriza por ser una persona informada, vitalmente activa y consciente de su identidad. Como individuos debemos tomar el papel como parte activa de una sociedad que requiere de seres humanos con posesión de conciencia histórica crítica para poder solventar las demandas de nuestro país y tomar decisiones de beneficio colectivo. 


\section{La Educación Indígena}

En el caso de la escuela indígena en la que se dio la experiencia que se describe, se habla una lengua propia de la región; como institución educativa, es sin duda es parte primordial de la educación en el Estado de Hidalgo. En la región del "Valle del Mezquital", se habla la lengua Hñähñu; dichas instituciones son coordinadas por la Dirección General de Educación Indígena, con el objetivo general de atender la política educativa nacional en materia de educación básica para la niñez indígena, se reconoce que este tipo de educación debe estar:

... Orientada a construir la unidad nacional, el
panorama educativo en las regiones indígenas
indicaba altas cifras de monolingüismo en sus
lenguas maternas, índices muy altos de
analfabetismo y de deserción, poca cobertura
educativa y escasa significación de la escuela entre
las expectativas de los pueblos indígenas. (Nolasco,
1997: 45)

Lo que nos lleva a pensar que no basta con ofrecer la enseñanza en la propia lengua indígena, sino que es necesario abordarla desde la complejidad, en el sentido de que debe partir desde sus referentes contextuales y abrir perspectivas sobre el medio indígena, y de esta manera fundamentar sus significaciones de lo que ha construido la educación indígena. En la actualidad también es menester hablar de la educación indígena dentro de un mundo globalizado, en el que los estándares curriculares nos brindan un panorama de los contenidos que deben aprender los alumnos de acuerdo al grado escolar en el que se encuentran, para ello la responsable es la Dirección General de Educación Indígena (DGEI), en virtud que es fue creada para normar, supervisar y evaluar los servicios escolarizados de escuelas indígenas en el nivel de preescolar y primaria, (DGEI, 2015).

Con respecto a la enseñanza de la Historia, dentro de las escuelas primarias indígenas, se imparte como parte del currículo. Cada maestro tiene un horario establecido en el que se encuentra la Historia como asignatura social y de formación ciudadana. Debe enseñarse una Historia que favorezca el desarrollo de los alumnos, tanto en los aspectos intelectuales como actitudinales, los cuales tienen que impactar al alumnado para aprender de manera continua sobre los acontecimientos que han enfrentado nuestras sociedades a lo largo del tiempo, los cuales nos han ayudado a recalcar nuestro papel como miembros pensantes de un devenir cotidiano.

\section{Experiencia de aprendizaje con el desarrollo del pensamiento complejo en la enseñanza de la Historia en la escuela primaria indígena}

El desarrollo del pensamiento complejo en la enseñanza de la Historia en escuela primaria indígena, conlleva a que los implicados en el proceso educativo (docentes), puedan proporcionar bases sólidas a los alumnos, mediante una buena planificación de las actividades que incluya la innovación. En este sentido, también es necesario contemplar las Tecnologías de la Información y Comunicación (TIC), porque no podemos quedar a la zaga de los medios digitales y del desarrollo tecnológico que está en gran parte del mundo. Para los niños estos medios son parte de la vida cotidiana. Respecto al pensamiento complejo, éste puede contribuir de manera significativa al logro de los aprendizajes esperados dentro del plan y programas de estudio de cada grado en la primaria, al desarrollar actividades que permitan ver a las partes como un todo.

La experiencia se aborda desde la investigación-acción, como medio de mejora en la práctica docente en la enseñanza y el aprendizaje de la Historia, mediante el uso de interactivos TIC, con la finalidad de acercar a los estudiantes de zonas indígenas a la sociedad de la información y comunicación. Así mismo, tener un espacio de recreación y de aprendizaje, al tratar la información histórica que se brinda dentro del aula. Se trabajó con 13 alumnos del grupo de quinto grado, en donde la innovación (como propuesta de diseño instruccional) se implementó, en la primer jornada de práctica en el semestre febrero- junio de 2018, en dos sesiones a la semana de 60 minutos cada una. Los niños usan la metodología del historiador para comprender la historia, desde el pensamiento complejo, con el apoyo de interactivos que permiten favorecer el aprendizaje. Entre los interactivos que se trabajaron, están los memoramas de personajes de la historia de México, así como crucigramas, mediante estos se trabaja parte de la historia de su entorno.

El objetivo general fue contribuir en el ámbito educativo, mediante el empleo de interactivos en la enseñanza de la historia con los alumnos, y el desarrollo del pensamiento complejo, al propiciar un clima innovador dentro de la clase, e identificar el resultado que se tiene con la aplicación de dichas actividades. La innovación, dentro de la práctica, se enmarca al usar interactivos realizados con TIC, como herramienta de la enseñanza en la clase de Historia, en virtud de que facilitan la transformación del niño pasivo en niño activo, siendo el protagonista del proceso de enseñanza y aprendizaje. Se despierta su curiosidad y sus ganas de saber más e investigar. Aquí, desde el pensamiento complejo, el uso de conceptos de 
primer y segundo orden en el aprendizaje de la Historia, adquieren importancia, pues hay una aproximación gradual en la construcción del conocimiento.

El grado de pensamiento complejo es el de: enseñar la comprensión, que de acuerdo con Escobar (2011), se puede dar en dos sentidos: a) la comprensión interpersonal e intergrupal y b) la comprensión a escala planetaria. En donde señala que Morin constató que la comunicación no implica comprensión; ésta siempre está amenazada por la incomprensión de los códigos éticos de los demás, de sus ritos y costumbres, de sus opciones políticas, señalando que los grandes enemigos de la comprensión son el egoísmo, el etnocentrismo y el sociocentrismo. Por tanto, enseñar la comprensión significa enseñar a no reducir el ser humano a una 0 varias de sus cualidades que son múltiples y complejas. De esta manera, la interactividad del niño abre caminos hacia una mejor comunicación con el docente y al analizar fuentes primarias y secundarias lo llevan a construir el conocimiento, lo que implica el análisis del contexto histórico y comprender la causalidad del suceso, de acuerdo al estudio de sus fuentes.

La Historia se hace más accesible al proporcionar información para analizar mayor variedad cultural, multitud de experiencias; por lo tanto, se desarrolla el pensamiento complejo, al usar sus competencias (emocionales, procedimentales y sociales) que amplían las destrezas del niño, y permiten comprender que un suceso está en interacción con diversos factores. De acuerdo con Zabala (1995), a lo largo de las diferentes etapas de la enseñanza obligatoria tenemos que diferenciar entre el proceso que sigue cada alumno y los resultados o competencias que va adquiriendo, por lo que importante identificar los tipos de conocimiento que va adquiriendo el niño a lo largo de su formación, pues no sólo son importantes los conocimientos fácticos, deben estar presentes también los procedimentales, los actitudinales, los cognitivos y los éticos. Esto principalemente en la enseñanza de la historia.

La institución en donde se realizó la experiencia de práctica tiene una sala de cómputo, con seis computadoras. Para la clase de Historia, se diseñaron actividades en la planificación " de quinto grado, correspondientes al Bloque IV, denominado "Del Porfiriato a la Revolución Mexicana". En dicho plantel se efectúan actividades permanentes en lengua indígena, para ello se realizó la traducción de conceptos de primer orden (suceso histórico) en lengua indígena. Antes de

\footnotetext{
La planificación de la clase se realizó tomando como base los seis pasos del diseño instruccional desde el pensamiento complejo, que fueron diseñados para la realización del proyecto RECREA, que enmarca estas actividades, de acuerdo a Van Merriënboer, J., J., G., y Kirschner P., A. (2010), Diez pasos para el aprendizaje complejo: Un acercamiento sistemático al diseño instruccional de los cuatro componentes.
}

iniciar la actividad, se presenta la rúbrica de evaluación, que considera el trabajo colaborativo y el logro de los aprendizajes esperados, en este caso los niños aportaron ideas para enriquecer la rúbrica.

Se inició con el concepto de primer orden, "Las diferencias políticas entre los liberales y la consolidación de la dictadura de Porfirio Díaz", se recuperan conocimientos previos, y el conflicto cognitivo a resolver, se da pauta para que los estudiantes planten sus hipótesis o supuestos en torno al tema, en grupo se realizan las preguntas de investigación para resolver el conflicto cognitivo; posteriormente, se investiga en diversas fuentes primarias (en ésta se recupera la actividad de preguntar a sus abuelos o bisabuelos, sobre algún relato propio de esa época), y en las instalaciones de la escuela secundaria, investigan en Internet (páginas confiables, observación de videos, visita a museos). Posteriormente, se les presentó el memorama: "Liberales y Conservadores", en el que se identificaron personajes, tales como: Benito Juárez, Nicolás Bravo, Porfirio Díaz, Vicente Guerrero, Antonio López de Santa Anna, Lucas Alamán y Juan Nepomuceno Almonte.

Después de investigar, se realizó un análisis a partir de los conceptos de segundo orden, mediante el uso de organizadores gráficos (cuadro comparativo de diferencias políticas entre liberales y conservadores), y se elabora el producto de la clase, que fue un cartel en el que se colocaron imágenes de los personajes identificados como liberales y conservadores. Se socializó el producto a partir de los ámbitos de la Historia, y finalmente, se utilizó un crucigrama para motivar y valorar los aprendizajes. Para llevar a cabo la evaluación de los resultados, se realizaron registros de observación dentro de la práctica en el espacio descrito, así como algunas entrevistas a los alumnos sobre lo aprendido con el uso de las TIC en la enseñanza de la Historia.

A partir de la observación se identificó que los estudiantes se apropiaron de diversos conocimientos en la investigación y al compartir con sus compañeros, se enriquece el conocimiento. Al analizar las entrevistas sobre las experiencias de la clase, los niños refieren que "les resultó interesante", "los motivó a investigar sobre la Historia y así poder entender mejor". Cuando ellos realizaron las diversas actividades con los interactivos, se dieron cuenta que también ellos son protagonista de la Historia. Mostraron interés de forma significativa, trabajaron colaborativamente, se vieron motivados al conocer la vida de algunos personajes, comprendieron el orden temporal de los acontecimientos que se suscitaron en un periodo de tiempo, por lo se fortalecen las competencias propias de la Historia en este grado educativo.

El uso de la rúbrica permite a los alumnos conocer los aspectos a evaluar, se motivan para trabajar y alcanzar 
niveles de desempeño e integrarse al trabajo colaborativo, y permitió que aportaran ideas para enriquecerla.

Las herramientas de TIC ofrecen información que debe ser empleada en el aula, el docente debe buscar y/o crear materiales que favorezcan el desarrollo del pensamiento Histórico en los niños.

Por lo expuesto hasta el momento, considero que esta experiencia propició el desarrollo del pensamiento complejo, porque los niños lograron obtener conocimientos basados en la experiencia, siguiendo los pasos del método científico. Con ayuda de la investigación, los niños buscaron las causas que originaron el suceso histórico, dieron una explicación del fenómeno comprendiendo en partes para así comprender el todo.

\section{Conclusiones}

Derivado del trabajo anterior, se puede concluir que la enseñanza de la Historia, a partir del pensamiento complejo, es una parte primordial dentro del aprendizaje, trae beneficios para la población indígena, se centran en la construcción de conocimiento en los alumnos; por ende, los docentes deben formar estudiantes reflexivos, competitivos e innovadores dentro de la sociedad actual. Al trabajar el pensamiento complejo, se desarrolla el pensamiento crítico y creativo; como docentes somos participes de la educación y debemos tomar en cuenta la enseñanza de la Historia, pues es de suma importancia. El niño conoce el por qué del presente, cómo se dieron los cambios a lo largo del tiempo, las causas y las consecuencias, así como los procesos que se siguieron en el desarrollo de ese fenómeno histórico. El presente resulta importante, como un punto de reflexión de lo ocurrido en el pasado que repercute en el momento histórico en el que nos desenvolvemos, al distinguir lo que nos afecta como sociedad, así como los rasgos significativos de una identidad propia que se forma en colectivo.

La enseñanza de la Historia desarrolla la identidad nacional. Mediante el conocimiento de ésta, el niño adquiere y desarrolla diversas competencias que favorecen su aprendizaje, al conocer los sucesos y personajes que intervinieron a lo largo de la Historia, para que en nuestro presente tengamos las condiciones en las que nos vemos envueltos. Si se logra desarrollar el pensamiento complejo en los estudiantes, mediante estrategias innovadoras, de manera concreta y sustancial, se posibilitará a los alumnos adquirir un pensamiento analítico y reflexivo para poder tomar las riendas de esta sociedad en la que nos vemos envueltos. Es necesario conocer la Historia para no estar condenados a repetirla. De igual manera, optar por aprender sobre Historia resulta favorable, nos abre veredas de la comunicación e interacción con terceros, dotándonos de ideas y pensamientos concretos que contribuirán de manera significativa en la toma de decisiones dentro de nuestra sociedad compleja y cambiante.

Nuestro deber como ciudadanos responsables es recuperar la Historia, dar pauta a reconocer su importancia y su sentido dimensional, ser críticos históricamente y obstaculizar la ceguera histórica. Considero que las TIC, en los últimos años, han repercutido significativamente en algunos planteles indígenas, porque han recibido equipos de cómputo y conexión a internet; esto, de alguna manera contribuye a mejorar las prácticas de enseñanza. Sin embargo, en muchos casos se reduce a la presentación de videos o investigar en internet, pero se olvida que la Historia permite a los niños despertar el interés o curiosidad por el pasado, desarrolla la capacidad de asombro y el pensamiento complejo permite ver al suceso histórico de manera integral, no fragmentado, ni aislado, comprender que éste es multicausal, porque está relacionado con los ámbitos: económico, político, social y cultural. Enseñar y aprender Historia remite a la reflexión constante a partir del contexto actual-cambiante y entender que existe una serie de interrelaciones que generan un mundo complejo.

\section{References}

Arteaga, Belinda y Camargo, Siddharta. (2014). "Educación histórica: una propuesta para el desarrollo del pensamiento histórico en el plan de estudios de 2012 para la formación de maestros de Educación Básica”. Revista Tempo e Argumento, Florianopolis, V.6, n.13, pp. 110-139. Septiembre/diciembre. Consultado junio, 2018. Disponible en http://www.revistas.udesc.br/index.php/tempo/article/viewFile/217518 0306132014110/4162

Berger, Peter L. y Luckmann, Thomas (1968). La construcción social de la realidad. Buenos Aires: Amorrortu (reimpresión 1998).

Bracho, Jorge (2009). "Historia, memoria y enseñanza". Revista de Teoría y Didáctica de las Ciencias Sociales. [En Línea] núm. 15, juliodiciembre, 2009, Universidad de los Andes Mérida, Venezuela. Disponible en línea en: http://www.redalyc.org/articulo.oa?id=65213215002

DGEI, (2015) Marco curricular de la Educación Inicial Indígena y de la población migrante. México, D. F: Secretaría de Educación Pública.

Escobar, M. Carolina. (2011). "El pensamiento complejo de Edgar Morin y los siete saberes necesarios para la educación del futuro". Gestiopolis, Núm. 21, octubre, 2011, Consultado marzo, 2018. Disponible en https://www.gestiopolis.com/pensamiento-complejoedgar-morin-saberes-necesarios-educacion-futuro/

Freire, Paulo (1997). Pedagogía de la indignación. Brasil: Paz y Tierra.

Lima Muñiz, Laura, Bonilla Castillo, Felipe y Arista Trejo, Verónica. (2010). "La enseñanza de la Historia en la escuela mexicana". Junio, 2018. Disponible en http://clio.rediris.es/n36/articulos/limaetalii.pdf 
Plá, Sebastián "La enseñanza de la historia como objeto de investigación" Secuencia, núm. 84, septiembre-diciembre 2012. Consultado en agosto 2018 Disponible

http://www.scielo.org.mx/pdf/secu/n84/n84a7.pdf

Morín, Edgar, (1994), Introducción al pensamiento complejo. España. GEDISA.

Nolasco, Margarita (1997). "Educación bilingüe: la experiencia en México". En Sánchez-Jaramillo, Luis Fernando. "La Historia como ciencia". Revista Latinoamericana de Estudios Educativos. Colombia En Línea vol. 1, núm. 1, julio-diciembre, 2005, Colombia: Universidad de Caldas, Manizales, Disponible en: http://www.redalyc.org/articulo.oa?id=134116845005

SEP (2011). Enseñanza y aprendizaje de la Historia en la Educación Básica. México, D. F: Secretaría de Educación Pública.

SEP (2011). Plan y Programa de Estudios. México, D. F: Secretaría de Educación Pública.

SEP (2017). Plan y Programa de Estudios para la educación básica. Aprendizaje Clave. Ciudad de México: Secretaría de Educación Pública.

Zabala, Antoni (1995). La práctica educativa. Cómo enseñar. España: Graó. Disponible en: https://des-for.infd.edu.ar/sitio/profesorado-deeducacion-inicial/upload/zavala-vidiella-antoni.pdf 